\title{
Multiple linear epitopes (B-cell, CTL and Th) of JEV expressed in recombinant MVA as multiple epitope vaccine induces a protective immune response
}

\author{
Fengjuan Wang ${ }^{1 \dagger}$, Xiuli Feng ${ }^{1 \dagger}$, Qisheng Zheng ${ }^{2}$, Hongyan Hou ${ }^{3}$, Ruibing Cao ${ }^{1 *}$, Bin Zhou', Qingtao Liu4,
} Xiaodong Liu', Ran Pang ${ }^{1}$, Jin Zhao ${ }^{1}$, Wenlei Deng ${ }^{1}$ and Puyan Chen ${ }^{1}$

\begin{abstract}
Epitope-based vaccination might play an important role in the protective immunity against Japanese encephalitis virus (JEV) infection. The purpose of the study is to evaluate the immune characteristics of recombinant MVA carrying multi-epitope gene of JEV (rMVA-mep). The synthetic gene containing critical epitopes (B-cell, CTL and Th) of JEV was cloned into the eukaryotic expression vector PGEM-K1L, and the rMVA-mep was prepared. BALB/c mice were immunized with different dosages of purified rMVA-mep and the immune responses were determined in the form of protective response against JEV, antibodies titers (IgG1 and IgG2a), spleen cell lymphocyte proliferation, and the levels of interferon- $y$ and interleukin-4 cytokines. The results showed that live rMVA-mep elicited strongly immune responses in dose-dependent manner, and the highest level of immune responses was observed from the groups immunized with $10^{7} \mathrm{TCID}_{50}$ rMVA-mep among the experimental three concentrations. There were almost no difference of cytokines and neutralizing antibody titers among $10^{7} \mathrm{TCID}_{50}$ rMVA-mep, recombinant ED3 and inactivated JEV vaccine. It was noteworthy that rMVA-mep vaccination potentiates the Th1 and Th2-type immune responses in dose-dependent manner, and was sufficient to protect the mice survival against lethal JEV challenge. These findings demonstrated that rMVA-mep can produce adequate humoral and cellular immune responses, and protection in mice, which suggested that rMVA-mep might be an attractive candidate vaccine for preventing JEV infection.
\end{abstract}

Keywords: Japanese encephalitis virus, rMVA-mep, Immune response, Protection response

\section{Introduction}

The Japanese encephalitis virus (JEV), which belongs to the family Flaviviridae, infects the human central nervous system [1,2]. The virus has a zoonotic transmission cycle between birds and mosquitoes, in which swine serves as an intermediate amplifier hosts and JEV can spread from swine to humans via mosquito bites [3-5]. Thus the vaccination of swine against JEV should contribute to minimize the occurrence of JE epidemics in humans [6].

\footnotetext{
* Correspondence: crb@njau.edu.cn

${ }^{\dagger}$ Equal contributors

'Key Laboratory of Animal Diseases Diagnosis and Immunology, Ministry of Agriculture, Nanjing Agricultural University, Nanjing 210095, China Full list of author information is available at the end of the article
}

Both inactivated and live-attenuated vaccines have been widely used in many Asian countries. Inactivated mouse brain vaccines are considered first generated, inactivated cell culture vaccines and live SA14-14-2 are considered second generation vaccines against JEV [7], which play vital roles on effectively decreased the morbidity of Japanese encephalitis. During the vaccination with both inactivated and live-attenuated JEV vaccines, there are various disadvantageous conditions, including poor availability, high production costs, poor long-term immunity, and the possibility of allergic reactions [8]. These problems may be solved by using epitope-based vaccines containing selected protective epitopes, appropriately presented, which are capable of stimulating effective $\mathrm{B}$ cell, $\mathrm{T}$ cell, and cytotoxic immune responses

\section{() Biomed Central}


while avoiding the induction of undesirable side-effects [9].

Judging from animal experiments, the protective immune response against JEV infection arises via both humoral and cellular immunity. Passive immunization with monoclonal antibodies specific for the JEV E protein has been shown to protect animals from lethal JEV challenge $[10,11]$ and the adoptive transfer of JEV-specific cytotoxic T lymphocytes (CTLs), which confer protection against lethal challenge in mice [12]. Likewise, the envelope (E) protein of JEV was reported to be a strong immunogen for the production of neutralizing antibodies and CTLs [13]. Many vital epitopes identified in the JEV E protein [14-16] are effective in inducing protective immunity against JEV. Moreover, it has been reported that the design and delivery of epitope-based vaccines have the potential to be a novel vaccine against JEV [17-19].

In recent years, modified vaccinia virus Ankara has showed great promise as vectors for recombinant vaccine development [20-24]. Despite its replication deficiency in human and most mammalian cells, MVA provides high-level gene expression and has been proven to be immunogenic when delivering heterologous antigens in animals and humans [25-27]. MVA vector vaccines induce significant levels of humoral and cellular immune responses to vaccine antigens and were found to be less affected by preexisting vaccinia virus-specific immunity when compared to replication-competent vaccinia virus vectors [28]. In this study, we first constructed the recombinant MVA by synthesizing multi-epitope gene from JEV envelope protein, which combined six B-cell epitopes (amino acid residues 75-92, 149-163, 258-285, 356-362, 373-399 and 397-403) and a CTL epitope (amino acid residues 60-68) and a Th epitope (amino acid residues 436-445) [29], and evaluated the abilities to induce immune responses in mice and protective efficacy against JEV challenge.

\section{Materials and methods}

\section{Cells and mice}

Baby hamster kidney (BHK-21) cell (ATCC CCL-10) and rabbit kidney cell (RK-13) cell (ATCC CCL-37) growed in Dubach's modified Eagle's medium (DMEM) supplemented with $10 \%$ heat-inactivated fetal bovine serum (FBS), $100 \mu \mathrm{g} / \mathrm{mL}$ of streptomycin, and $100 \mu \mathrm{g} / \mathrm{mL}$ of penicillin.

Female BALB/c mice (4-6 weeks old) were purchased from the Experimental Animal Center of Nanjing Medical University (Nanjing, China). All animal experiments were conducted according to the guidelines approved by the Animal Ethical and Experimental Committee of Nanjing Agriculture University.

\section{Viruses and vaccination control}

The SA14 strain of JEV, which maintained in our laboratory, was propagated in BHK-21 for the plaque reduction neutralization test (PRNT) and challenge test. The viral titers of the supernatants were approximately $6.3 \times 10^{7} \mathrm{pfu}$.

MVA (582nd CEF passage), which maintained in our laboratory, was propagated in BHK-21 and its viral titers of the supernatants were approximately $1.0 \times 10^{8} \mathrm{pfu}$.

Recombinant proteins MEP and EDIII(which were used as vaccination control)were expressed in $E$. coli BL21 as previous reported [30], and purified on $\mathrm{Ni}$ affinity chromatography column (Amersham Bioscience HiTrap chelating HP $5 \mathrm{~mL} \times 1$ column) according to the manufacturer's instructions.

The inactivated JEV vaccine (SA14-14-2 strain, $2.0 \times 10^{7} \mathrm{pfu}$ ) was obtained from ZHONGMU BIOINDUSTRY CO., LTD.

\section{Preparation of rMVA-mep Construction of the rMVA-mep}

In this paper, according to the previously report [29], the multiple-epitope fragment from the $\mathrm{E}$ protein of JEV (SA14-14-2 strain), named MEP (eight epitopes), was designed by arranging the eight epitopes in the order of amino acids (75-92)-(149-163)-(258-285)-(356-362)(373-399)-(397-403)-(60-68)-(436-445). The amino acid sequence and the nucleotide sequence of MEP are shown in Figure 1A. To minimize interference between adjacent epitopes, each was separated from its neighboring epitope by a glycine and a serine codon [29]. The multiple-epitope gene was chemically synthesized by Invitrogen Biotechnology Co. Ltd. (Shanghai, China) and cloned into the transfer vector pGEM-K1L plasmid and named pGEM-K1L-mep (Figure 1B).

The MVA recombinants were produced according to the manufacturer's instructions [31] on BHK-21 cells, named rMVA-mep-BHK-21. Simple, the rMVA-mepBHK-21, which included rMVA-mep and wild MVA, was purified by serially infecting RK-13 cells, which was called rMVA-mep-RK-13. The rMVA-mep-RK-13 with k1l gene but no MVA was used to transfect BHK-21 cells, in which k1l was removed by intra-genomic homologous recombination. The purified recombinant MVA containing multiple-epitope gene was called rMVA-mep, which was determined by the tissue culture infectious dose $50\left(\mathrm{TCID}_{50}\right)$ methods.

\section{Identification of rMVA-mep by $P C R$}

To identify that the rMVA-mep contains targeted gene MEP, the genome of RK-13 cells infected with recombinant viruses were prepared, and PCR was used with the specific primers of the targeted gene MEP, and specific gene of wild MVA. Also, the genome of BHK-21 cells 
A GAA TTC CCG ACC ACC GGC GAA GCG CAT AAC GAA AAA CGT GCG GAT AGC AGC TAT GTG TGC

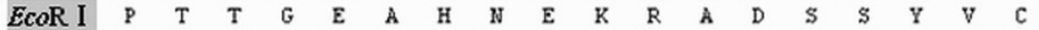
CTT AAG GGC TGG TGG CCG CTT CGC GTA TTG CTT TTT GCA CGC CTA TCG TCG ATA CAC ACG

GGC TCT AGC GAA AaC CAT GGC AAC TAT AGC GCA CAG GTG GGT GCA AGC CAG GGT AGC CAG

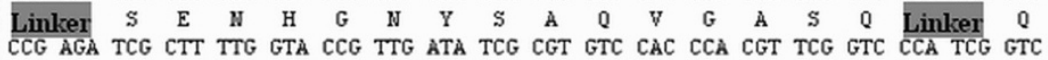

GAA GGT GGT CTG CAT CAG GCA CTG GCA GGT GCA ATC GTG GTG GAA TAT AGC ÄC AGC GTG

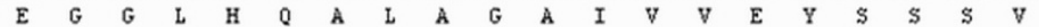
CTT CCA CCA GAC GTA GTC CGT GAC CGT CCA CGT TAG CAC CAC CTT ATA TCG TCG TCG CAC

AAA CTG ACC AGC GGT CAT CTG GGT AGC ACC GTG AAC CCG TTT GTG GCA GGT AGC GAA ATG $\begin{array}{llllllllllllllllll}\text { K } & \text { L } & \text { T } & \text { S } & \text { G } & \text { H } & \text { L } & \text { Linker } & T & \text { V } & \text { N } & \text { P } & \text { F } & \text { V } & \text { A } & \text { Linker } & \text { E } & \text { M }\end{array}$ TTT GAC TGG TCG CCA GTA GAC CCA TCG TGG CAC TTG GGC AAA CAC CGT CCA TCG CTT TAC

GAA CCG CCG TTT GGÂ GAT AGC TAT ATC GTG GTG GGC CGT GGC GAC AAA CAG ATC AAC CAC $\begin{array}{llllllllllllllllllllll} & E & P & P & F & G & D & S & Y & I & V & V & G & R & G & D & K & 0 & I & N & H\end{array}$ CTT GGC GGC AAA CCT CTA TCG ATA TAG CAC CAC CCG GCA CCG CTG TTT GTC TAG TTG GTG

CAC TGG CAT AAA GCG GGC AGC CAT AAA GCG GGC AGC ACC CTG GGT TCT TGC TAT CAT GCA

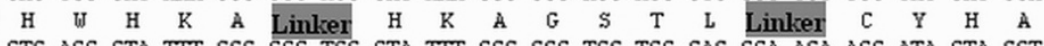
GTG ACC GTA TTT CGC CCG TCG GTA TTT CGC CCG TCG TGG GAC CCA AGA ACG ATA GTA CGT

AGC GTG ACC GAT ATC GGT TCT AGC ATC GGC CGT GCG GTG CAT CAG GTG TTT TAA CTC GAG

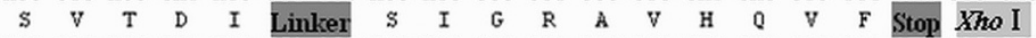
TCG CAC TGG CTA TAG CCA AGA TCG TAG CCG GCA CGC CAC GTA GTC CAC AAA ATT GAG CTC

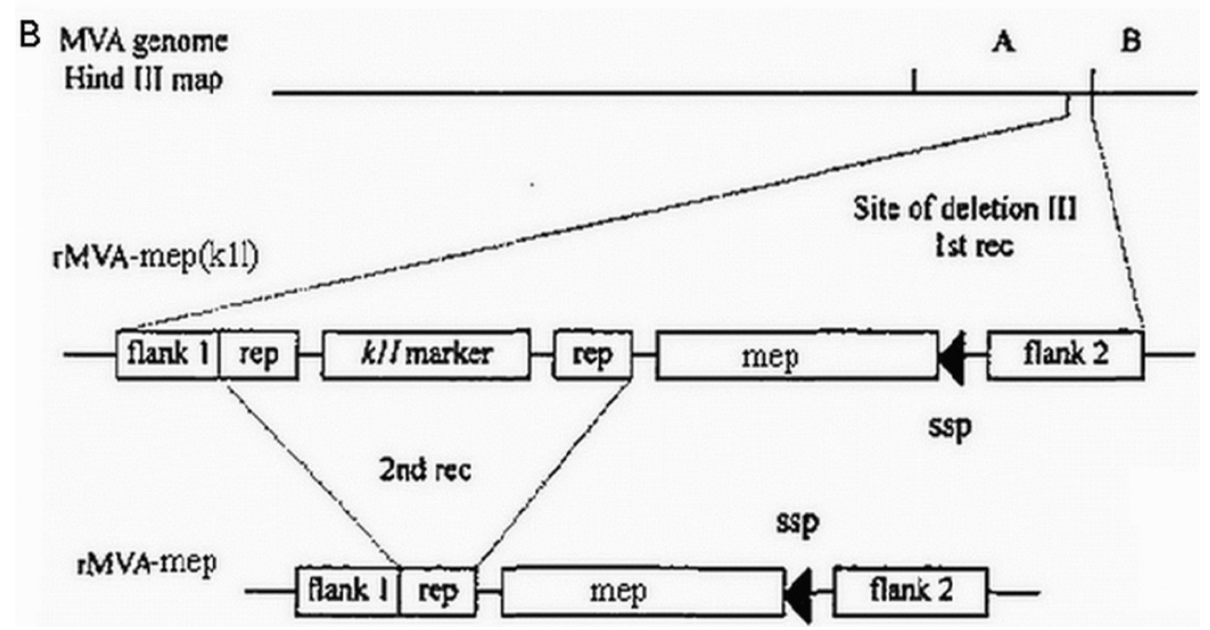

Figure 1 Construction of the rMVA-mep. A. Design and construction of the multi-epitope peptide (MEP). The MEP was constructed from six B-cell epitopes and two T-cell epitopes, with a glycine and a serine (GS) as a spacer between epitopes. The amino acid sequences of the epitopes were obtained from the envelope protein of the JEV (AF315119) [29]. B. The construction of the rMVA-mep. pGEM-K1L-mep contains the multiepitope peptide (MEP).

infected with recombinant viruses were prepared to detect the host range gene k1l and MEP by PCR method. These primers used were shown in Table 1.

\section{Identification of expressed proteins of MEP}

Western blot was performed as described previously [29]. The lysates of BHK-21 cells infected with or without rMVA-mep were separated by $15 \%$ SDS-PAGE, in which non-infected BHK-21 cells was used as a negative control. The expressions of MEP proteins were identified
Table 1 The primers of Identification of rMVA-mep by PCR

\begin{tabular}{ll}
\hline Gene & Primer (from $\mathbf{5}^{\prime}$ to $\mathbf{3}^{\mathbf{\prime}}$ ) \\
\hline Mep & Forward primer: ctcgagatgccgaccaccggcgaagcgca \\
& $\begin{array}{l}\text { Reverse primer: } \\
\text { catatgatttttataaaaatttaaaacacctgatgcaccgcacggccgatgctag }\end{array}$ \\
\hline K11 & Forward primer: gaatgcacatacataagtaccggcatctctagca \\
& Reverse primer: caccagcgtctacatgacgagcttccgagtt \\
\hline MVA (wild) & Forward primer: acataagtaccggcatctctagcacacagc \\
& Reverse primer: ttcgccggtggtcggcatctcgagagctac \\
\hline
\end{tabular}


using JEV-positive serum (PRIONCS, Switzerland) by West-Blotting analysis.

\section{Mouse immunization}

Mice were randomly divided into eight experimental groups (fifteen mice each group) and were immunized intraperitoneally (i.p.) on weeks 0,2 and 4, respectively. The Group 1, Group 2 and Group 3 were given the $10^{6}$ $\mathrm{TCID}_{50} / 0.1 \mathrm{~mL}, 10^{7} \mathrm{TCID}_{50} / 0.1 \mathrm{~mL}$ and $10^{8} \mathrm{TCID}_{50} / 0.1 \mathrm{~mL}$ rMVA-mep, respectively. Vaccine group mice were each given $0.1 \mathrm{ml}$ of JEV (SA14-14-2 strain, $2 \times 10^{6} \mathrm{pfu}$ ). Also, the recombinant protein immunization groups were given

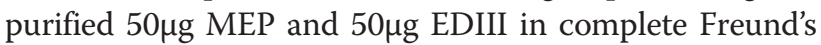
adjuvant (CFA) for the first immunizations, in incomplete Freund's adjuvant (IFA) for the second and the third immunizations. In addition, mice immunized with $0.1 \mathrm{~mL}$ MVA $\left(2 \times 10^{6} \mathrm{pfu}\right)$ or $0.1 \mathrm{~mL}$ PBS was used as negative controls (Table 2), respectivley. One week after the final immunization, three mice from each group were sacrificed, and their spleens removed aseptically for in vitro lymphocyte proliferation assay. Also, on $14^{\text {th }}$ day after the third immunization, mice were challenged by i.p. injection of a lethal dose of $5 \times 10^{6} \mathrm{pfu}$ of the JEV (SA14 strain) and the survival of the mice was monitored daily up to 15 days post-challenge. The animal groups and immunization doses were described in Table 2, and immunization schedule was shown in Figure 2.

\section{Antibody assays}

The sera were collected at 14, 28 and 42 days after immunization to perform the antibody assay by ELISA. Simply, the purified multi-epitope protein (rMEP) was coated in 96 -well plates $(1 \mu \mathrm{g} / \mathrm{mL})$ overnight at $4^{\circ} \mathrm{C}$. After blocking, the coated plates were incubated with mouse serum in a sequential dilution of 1:50, 1:100, 1:200, 1:400, $1: 800,1: 1600,1: 3200$ and $1: 6400$ for $1.5 \mathrm{~h}$ at $37^{\circ} \mathrm{C}$. And then, $50 \mu \mathrm{L}$ of a 1:1000 dilution of HRP- conjugated goat anti-mouse IgG, and a 1:2000 dilution of IgG1 and IgG2a were incubated for $45 \mathrm{~min}$ at $37^{\circ} \mathrm{C}$. After extensive washing, tetramethyl benzidine (TMB) substrate was added for 10 minutes at room temperature, and the reaction was stopped with $2 \mathrm{M} \mathrm{H}_{2} \mathrm{SO}_{4}$, and absorbance was read at $450 \mathrm{~nm}$. The titer was expressed as the highest serum dilution giving an absorbance $>0.2$. The background value was 0.061 , and 0.2 was used as cut-off. Each serum titration was assayed in triplicates.

\section{Estimation of IFN- $\gamma$ and IL-4 by ELISA}

At $7^{\text {th }}$ day after the final immunization, the sera were collected from all experiment groups to measure the productions of IL- 4 and IFN- $\gamma$ using commercially mice cytokine ELISA kits (RD, USA), according to the manufacturer's instructions.

\section{Lymphocyte proliferation assay}

One week after the final immunization, three mice of each group were killed, and splenocyte were prepared, and were incubated with $10 \mu \mathrm{g} / \mathrm{mL} \mathrm{rMEP}$ protein in 96-well flat-bottomed microtiter plates $(100 \mu \mathrm{L} /$ well, $2 \times 10^{6}$ cells $/ \mathrm{mL}$ in RPMI 1640 medium with $10 \% \mathrm{FBS}$ ). After incubation for $72 \mathrm{~h}$, a final concentration of $20 \mu \mathrm{g} / \mathrm{mL}$ MTT (Sigma) was added to each well for $4-\mathrm{h}$ incubation. The medium was removed, and $100 \mu \mathrm{L}$ of DMSO was added into each well [32]. The light absorbance was measured at $570 \mathrm{~nm}$ to analyze cell viability expressed as the value of the A570 of cells from immunized mice vs. the control samples. All the data were statistically analyzed with SPSS software.

\section{Plaque reduction neutralization assay (PRNT)}

Neutralization antibodies elicited in immunized mice were valuated by PRNT as described previously [33]. Mouse serum was heat inactivated at $56^{\circ} \mathrm{C}$ for $30 \mathrm{~min}$. Twofold serial dilutions of murine sera starting at 1:5 were tested, and then incubated with $100 \mathrm{pfu}$ of JEV (virulent SA-14 strain) at $37^{\circ} \mathrm{C}$ for $1 \mathrm{~h}$. The virus titers were then determined by plaque formation on BHK21cell monolayers [34,35]. Percentage neutralization was calculated from the number of plaques obtained in the presence and the absence of the serum. The reciprocal of the highest serum dilution giving at least 50\%

Table 2 The diagram of mouse immunization

\begin{tabular}{|c|c|c|c|c|}
\hline \multicolumn{2}{|l|}{ Groups } & \multirow{2}{*}{$\frac{\text { Priming }}{10^{6} \mathrm{TCID}_{50} / 0.1 \mathrm{~mL}}$} & \multirow{2}{*}{$\frac{\text { Boost } 1}{10^{6} \mathrm{TCID}_{50} / 0.1 \mathrm{~mL}}$} & \multirow{2}{*}{$\frac{\text { Boost } 2}{10^{6} \mathrm{TCID}_{50} / 0.1 \mathrm{~mL}}$} \\
\hline rMVA-mep & $10^{5} \mathrm{TCID}_{50}$ & & & \\
\hline & $10^{6} \mathrm{TCID}_{50}$ & $10^{7} \mathrm{TCID}_{50} / 0.1 \mathrm{~mL}$ & $10^{7} \mathrm{TCID}_{50} / 0.1 \mathrm{~mL}$ & $10^{7} \mathrm{TCID}_{50} / 0.1 \mathrm{~mL}$ \\
\hline & $10^{7} \mathrm{TCID}_{50}$ & $10^{8} \mathrm{TCID}_{50} / 0.1 \mathrm{~mL}$ & $10^{8} \mathrm{TCID}_{50} / 0.1 \mathrm{~mL}$ & $10^{8} \mathrm{TCID}_{50} / 0.1 \mathrm{~mL}$ \\
\hline \multicolumn{2}{|c|}{ Inactivated JEV vaccine } & $0.1 \mathrm{~mL}\left(2 \times 10^{6} \mathrm{pfu}\right)$ & $0.1 \mathrm{~mL}\left(2 \times 10^{6} \mathrm{pfu}\right)$ & $0.1 \mathrm{~mL}\left(2 \times 10^{6} \mathrm{pfu}\right)$ \\
\hline \multicolumn{2}{|l|}{ EDIII } & $50 \mu \mathrm{g} C F A$ & $50 \mu \mathrm{g}$ IFA & $50 \mu \mathrm{g}$ IFA \\
\hline \multicolumn{2}{|l|}{ rMEP } & $50 \mu \mathrm{g} C F A$ & $50 \mu \mathrm{g}$ IFA & $50 \mu \mathrm{g}$ IFA \\
\hline \multicolumn{2}{|l|}{ PBS } & $0.1 \mathrm{~mL}$ & $0.1 \mathrm{~mL}$ & $0.1 \mathrm{~mL}$ \\
\hline \multicolumn{2}{|l|}{ Wild MVA } & $0.1 \mathrm{~mL}\left(2 \times 10^{6} \mathrm{pfu}\right)$ & $0.1 \mathrm{~mL}\left(2 \times 10^{6} \mathrm{pfu}\right)$ & $0.1 \mathrm{~mL}\left(2 \times 10^{6} \mathrm{pfu}\right)$ \\
\hline
\end{tabular}

CFA, complete Freund's adjuvant; IFA, incomplete Freund's adjuvant. 


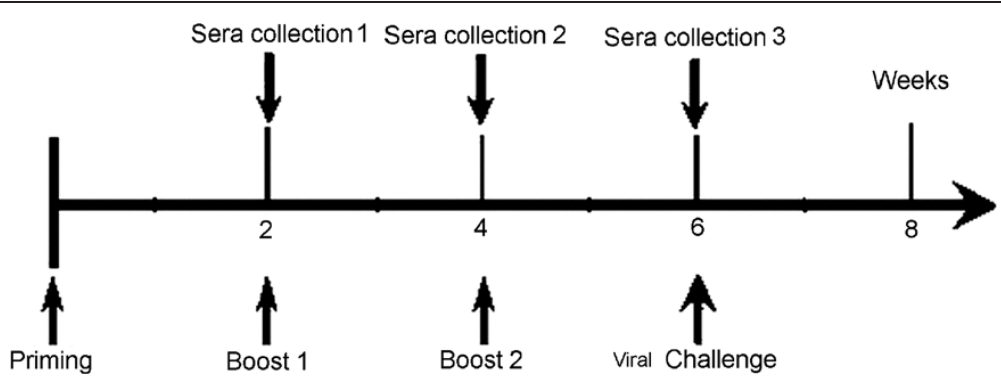

Figure 2 Vaccination schedule and viral challenge period of in vivo experiment. Mice were given a first followed by a second immunization and third immunization. The sera samples were collected at 14, 28 and 42 days after immunization. The immunized mice were challenged intraperitoneally with a lethal dose of $5 \times 10^{6} \mathrm{pfu}$ of the JEV (SA14 strain). The health of each mouse was assessed twice daily.

neutralization was regarded as the JEV neutralization titer.

\section{Statistical analysis}

The data were recorded as mean \pm standard deviation (SD). Biochemical and physiological parameters were analyzed statistically using one-way analysis of variance (ANOVA) followed by Dunnet- $t$-test using SPSS statistical software to evaluate variations between groups. A value of $\mathrm{P}<0.05$ was considered to be statistically significant.

\section{Results}

\section{Generation of the recombinant virus (rMVA-mep) and} purification

Recombinant viruses carrying wild MVA and host range gene k1l were selected by alternately infecting RK-13 cells and BHK-21cells. The first generation of purified viruses included targeted gene MEP (437bp), specific gene of wild MVA (nearly 700bp) and K1l gene (1332bp) (Figure 3A, lane 4: RK-13-1). To contract with the first generation, the concentration of wild MVA of the fourth generation, RK-13-4 (Figure 3A, lane 2), was obviously lower. There was no wild MVA in the last generation (RK-13-6, Figure 3A, lane 1 and Figure 3B, lane 8), and only the targeted recombinant viruses carrying host range gene k1l (Figure 3B, lane 6) and targeted gene MEP (Figure 3B, lane 7) were presented. Under nonselective growth conditions, k1l was removed by intragenomic homologous recombination when the recombinant viruses (RK-13-6) were reinfected into BHK-21 cells (Figure 3C). K1l could not be found in the six generation on BHK-21 cells (Figure 3C, lane 10), but not in the fourth, second and first generation (Figure 3C, lane11: BHK-21-4; lane12: BHK-21-2; lane13: BHK-21-1). These results showed that recombinant virus has been purified.

\section{Western Blotting of rMVA-mep}

The rMEP was highly expressed in E. coli, and purified with the expected $17.9 \mathrm{kDa}$ protein verified by Western blotting analysis (Figure 3D, lane 1). Moreover, it was observed that the MEP of JEV was stably expressed in BHK-21 cells after rMVA-mep infection, which were proved by Western blotting analysis with the sixth

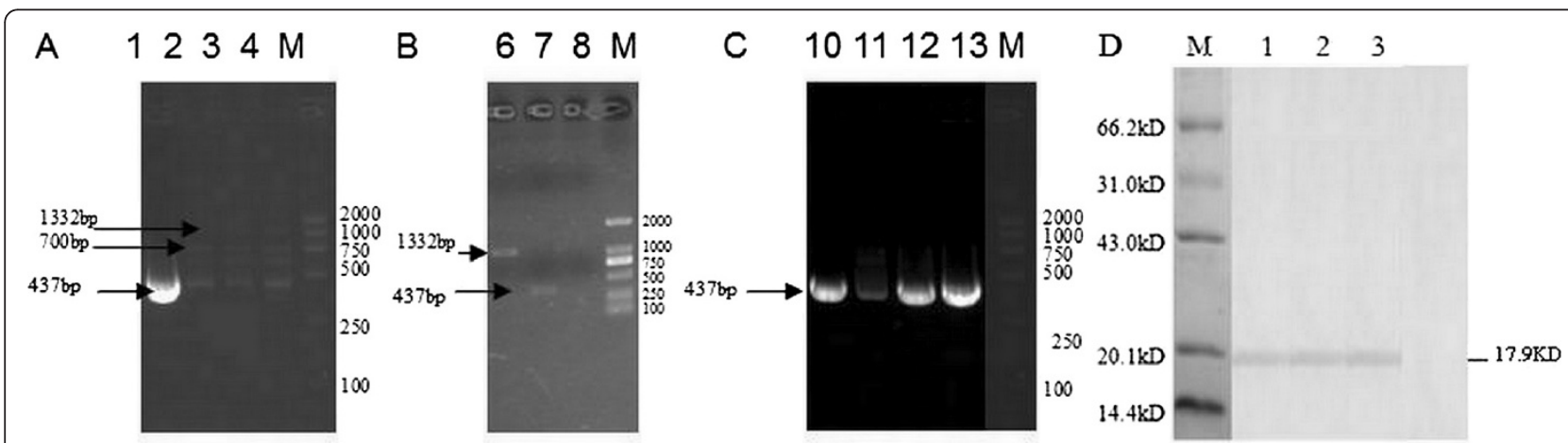

Figure 3 Identification of rMVA-mep. (A, B, C) Identification of rMVA-mep by PCR using primers targeted to MEP, to k1I and to MVA gene. (A) Lane 1, 2, 3, 4: infected cell focus RK-13-6, RK-13-4, RK-13-3, and RK-13-1, respectively. M: DL2000 marker. (B) Lane 6, 7, 8: infected cell focus RK-13-6(k1l gene), RK-13-6(MEP gene), and RK-6(no wild MVA), respectively. M: DL2000 marker. (C) Lane 10, 11, 12, 13: infected cell focus BHK-21-6, BHK-21-4, BHK-21-2, and BHK-21-1, respectively. M: DL2000 marker. (D) Western Blot analysis of rMVA-mep infected BHK-21. M: Low molecular protein marker; lane 1: Western blot of purified rMEP in E. coli. Lane 2: Western blot of purified rMVA-mep infected BHK-21-6 (The sixth generation on BHK-21 cells). Lane 3: Western blot of purified rMVA-mep infected BHK-21-16. Lane 4: BHK-21 cells. 
generation of rMVA-mep-infected BHK-21 cells and the sixteenth generation of rMVA-mep-infected BHK-21 cells (Figure 3D, lane 2 and lane 3). No $17.9 \mathrm{kDa}$ protein was found in the negative control of BHK-21 cells (Figure 3D, lane 4). These results demonstrated that the MEP gene was successfully expressed in the rMVA-mep with genetic stability and good immunogenicity.

\section{Cellular immune responses}

Cellular immune responses were evaluated by measuring the production of IFN- $\gamma$ and IL-4 by splenocytes from mice at $35 \mathrm{dpi}$ when stimulated by rMEP protein. As shown in Figure 4A, IFN- $\gamma$ and IL-4 productions from mice received rMVA-mep $\left(10^{5} \mathrm{TCID}_{50}, 10^{6} \mathrm{TCID}_{50}\right.$, $10^{7} \mathrm{TCID}_{50}$ ), inactivated vaccine, EDIII, and rMEP were significantly higher than that in the PBS and wild MVA $(\mathrm{P}<0.05)$. Mice immunized with rMVA-mep promoted the IFN- $\gamma$ and IL-4 levels in dose-dependent manner, in which cytokines production of mice immunized with $10^{7} \mathrm{TCID}_{50}$ rMVA-mep was observed highest among the three dosages. Also, the levels of IFN- $\gamma$ and IL-4 were similar among mice immunized with rMVA-mep $\left(10^{7} \mathrm{TCID}_{50}\right)$, inactivated vaccine, EDIII, and rMEP. These results suggested that rMVA-mep could induce both Th1 and Th2 type immune response.

The level of lymphocyte proliferation varied among the groups as shown in Figure 4B. The highest occurred in the group immunized with inactivated vaccine of JEV SA14-14-2, and this level was significantly higher than those produced by PBS, wild MVA, or rMVA-mep $\left(10^{5} \mathrm{TCID}_{50} ; 10^{6} \mathrm{TCID}_{50}\right)$. We also found that the level of splenocyte proliferation observed from the groups immunized with rMVA-mep $\left(10^{7} \mathrm{TCID}_{50}\right)$, EDIII, and rMEP was nearly equal; rMVA-mep can enhance antigen-specific splenocyte proliferative response in a dose-independent manner. No significantly lymphocyte

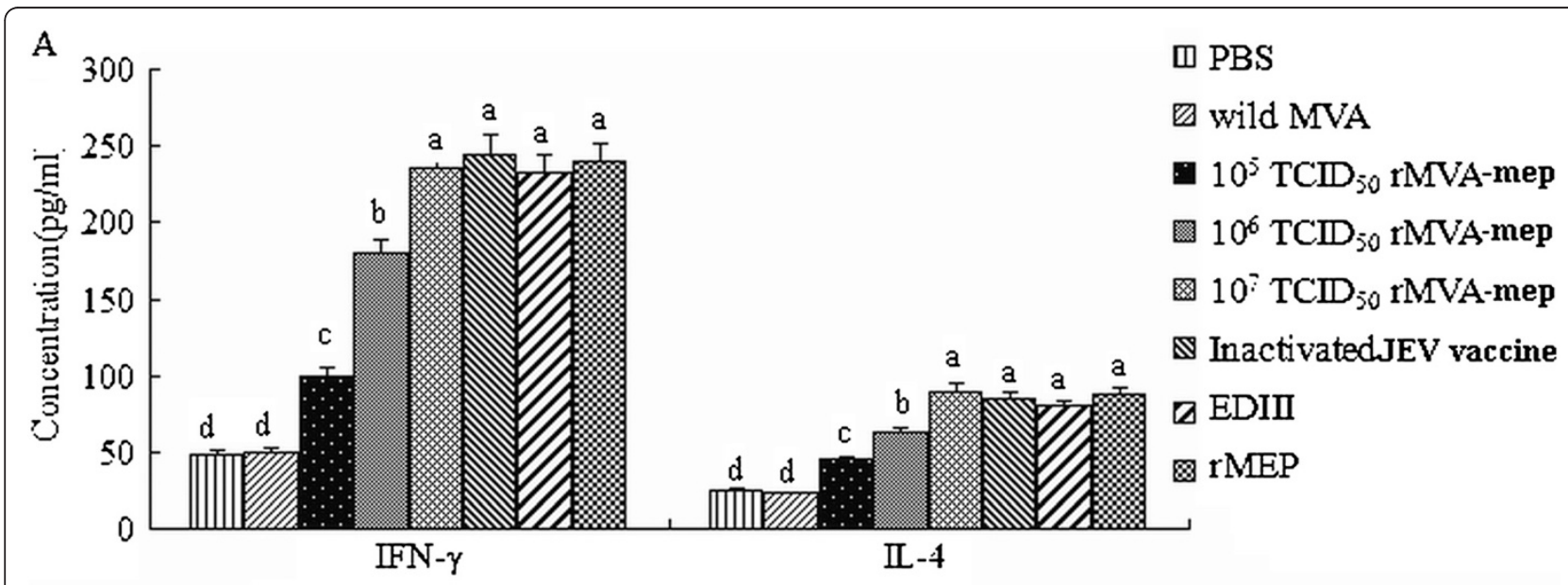

B

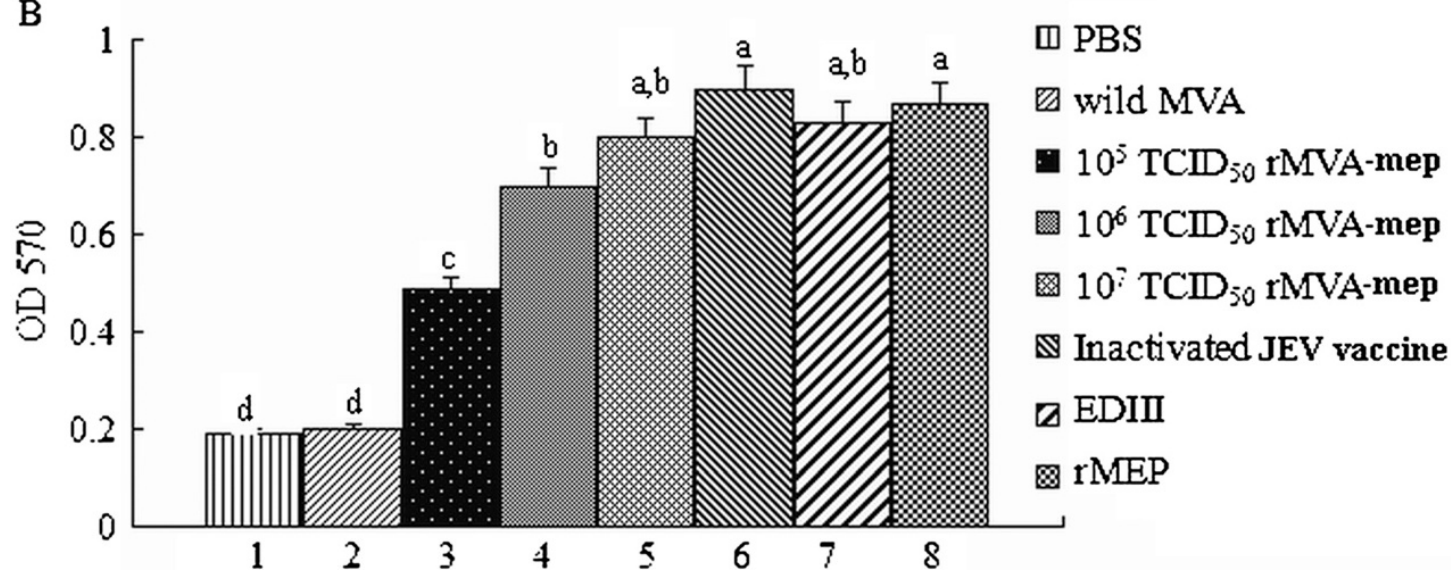

Figure 4 The cellular-mediated immune responses. (A) The cytokines. After $2^{\text {nd }}$ boost immunized 1 week, the sera from all experiment groups were collected to detect cytokine by ELISA. (B) spleen lymphocyte proliferation response. The spleen lymphocytes were incubated in 96-well flatbottomed microtiter plates and stimulated with rMEP for detecting the spleen lymphocyte proliferation response by MTT. The data are shown as mean \pm SD of three independent experiments. Statistically significant differences $(P<0.05)$ are indicated with different small letters. 
proliferation was induced by immunization with PBS and wild MVA.

\section{Anti-rMEP of JEV antibody titers and subtype analysis}

To estimate the potential roles of rMVA-mep on the humoral immune response, the IgG isotopes profiles of sera from immunized mice at two weeks after three immunizations were measured by ELISA method (Figure 5). These results showed that mice immunized with rMVA-mep generated a significant rMEP-specific antibody response in dose-dependent manner, in which mice injected with $10^{7}$ TCID50 rMVA-mep produced the strongest antibody response among the three reachable dosages (Figure 5A, IgG). Also, it was observed that the level of IgG from mice given the inactivated vaccine, EDIII and MEP were higher than that of mice given with $10^{7}$ TCID50 rMVA-mep. It was found that a combined IgG1/2a response was characteristic of mice immunized using an rMVA-mep prime-boost protocol (Figure 5B, IgG1 and Figure 5C, IgG2a). Moreover, the antibody level of IgG1 was significantly higher than that of IgG2a, which indicated that the IgG1 antibody was the dominant antibody subtype induced by rMVA-mep vaccination. Low levels of both IgG1 and IgG2a were found in control sera.

These results suggested that rMVA-mep might be as potential candidate vaccine for the prevention of JEV infection.

\section{Neutralizing antibodies}

Serum samples collected on days 14, 28 and 42 were further evaluated for their ability to neutralize JEV in vitro by the plaque reduction neutralization assay (PRNT). As shown in Table 3 ( $2^{\text {nd }}$ boost), although neutralization titer of serum from mice immunized with rMVA-mep $\left(10^{5}\right.$ TCID50 $[20 \pm 1.33]$ and $10^{6}$ TCID50 [33 \pm 2.33$])$ was significantly lower than that of the mice immunized with JEV vaccine $(58 \pm 2.01)$, EDIII $(56 \pm 1.99)$ and MEP $(57 \pm 1.99)$, rMVA-mep vaccination $\left(10^{7} \mathrm{TCID}_{50}[56 \pm 1.01]\right)$ almostly produced the equal level of neutralization antibody to that JEV vaccine, EDIII and MEP vaccinations. No JEV-neutralizing antibodies were found in the PBS or wild MVA control group.

\section{Mouse protection against JEV challenge}

To investigate the development of protective immunity further, all immunized mice were intraperitoneally challenged with a lethal dose $\left(5 \times 10^{6} \mathrm{pfu}\right)$ of JEV SA14 at two weeks after the final immunization. After the challenge, mice immunized with rMVA-mep $\left(10^{7} \mathrm{TCID}_{50}\right)$, EDIII, and rMEP or the inactivated vaccine showed complete $(100 \%)$ protection against JEV challenge, but only one mouse (1/12) survived in the PBS control group. Mice of the wild MVA group almost no survived (Table 3).

\section{Discussion}

It was reported that the recombinant multi-epitope peptide (rMEP) from JEV using epitope-based vaccine strategies is capable of inducing remarkable humoral and cellular immune responses and provided complete protection against lethal JEV challenge in mice [29]. Modified vaccinia virus Ankara (MVA) has been used to develop recombinant vaccines against the vital diseases including JEV, with some advantages, including contributing to replication-deficient virus efficiently replicates, high bio-safety, high-level immunogenic heterologous antigens expression. So it has been wildly used to develop genetic engineering vaccines and gene therapy [28]. In this paper, we investigated the potential immune functions of the recombinant multi-epitope peptide (rMEP) from JEV expressed in recombinant MVA as multiple epitope vaccine.

The E protein of JEV is a major immunogenic antigen, which shares various B-cell and T-cell epitopes [36]. Some studies have shown that fusion proteins containing Th epitopes may enhance antibody production against the B cell determinant [37], and the linking of a Th epitope to a CTL determinant is effective in the generation of antiviral CTLs [38]. Moreover, the Th cells activated by a vaccine that contains CD4+ Th epitopes may secrete several CTL-inducing or antiviral cytokines, induce CTL responses, and maintain CTL memory [39]. Ideally, epitope-based vaccines should contain both B-cell and T-cell epitopes (CTL epitopes, Th epitopes) that will serve to induce humoral and cellular immune responses. Therefore, in the present study, the selected epitopes containing six B-cell epitopes $\{(75-92)-(149-163)-$ (258-285)-(356-362)-(373-399)-(397-403)\}, one CTL epitope (60-68), and one Th epitope (436-445) from the E protein of JEV [29] was constructed and generated the recombinant virus (rMVA-mep), designated rMVA-mep. In this paper, we obtained a recombinant MVA expressing recombinant multi-epitope peptide (rMEP) from JEV with genetic stability and good immunogenicity (Figure 3D).

Balb/c mice are widely used experimental animal models. The immune epitopes and protection functions of JEV are verified on Balb/c mice immunization experiments [29]. Therefore, in this paper, we select the Balb/c mice model to confirm the immune response of multiple linear epitopes (B-cell, CTL and Th) of JEV expressed in recombinant MVA. Although the results of Figures 4 and 5 showed no difference of IFN- $\gamma$, IL-4, IgG1, and IgG2a titers among $10^{7} \mathrm{TCID}_{50}$ rMVA-mep, recombinant ED3 and inactivated JE vaccine, it was also found that live rMVA-mep elicited strongly immune responses 


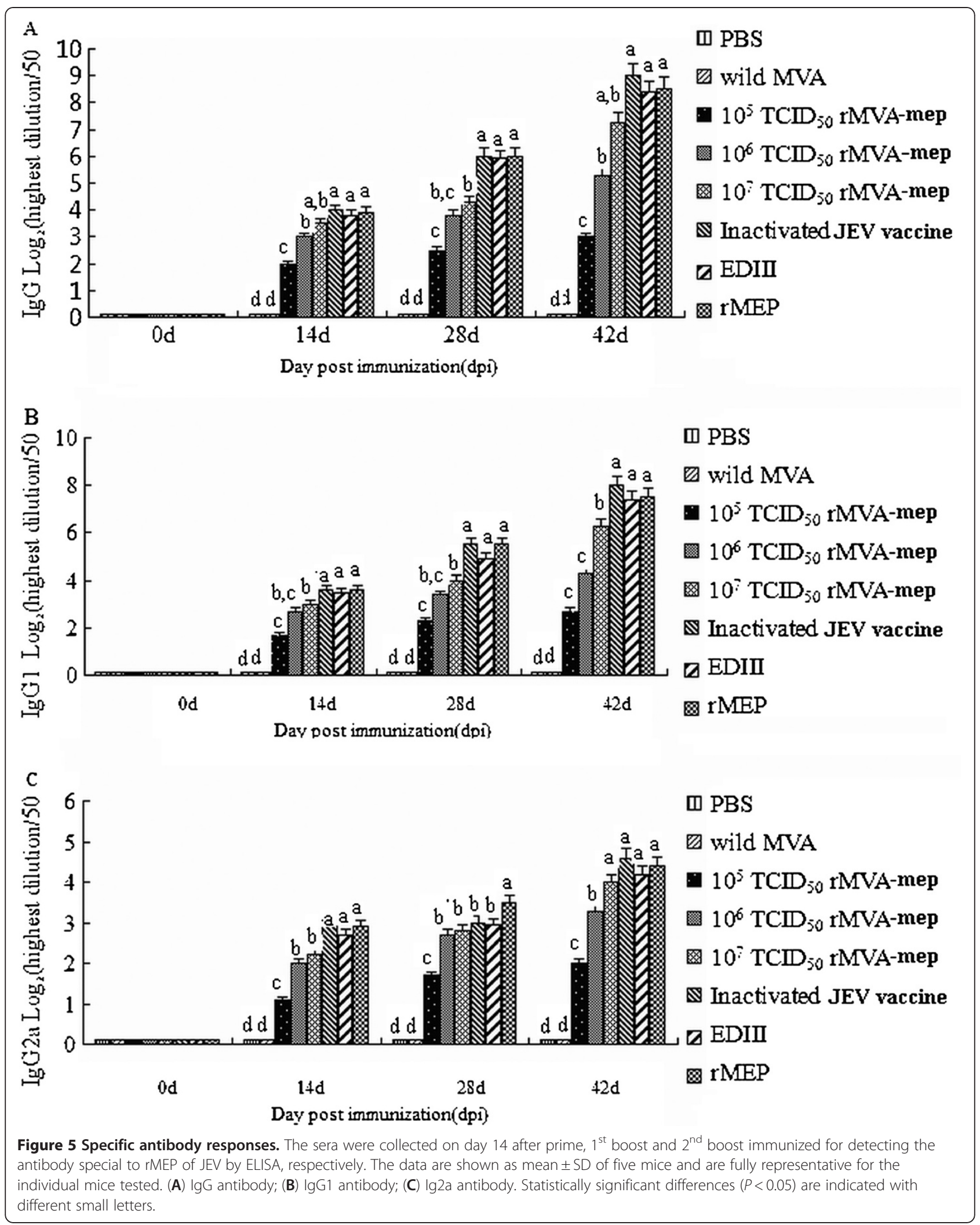


Table 3 The neutralizing antibody levels and protection against JEV infection in immunized mice

\begin{tabular}{llllll}
\hline Treatment & & \multicolumn{2}{l}{ PRNT50 $^{\mathbf{a}}$} & \multicolumn{2}{l}{$\begin{array}{l}\text { Survival } \\
\text { rate (\%) }\end{array}$} \\
\cline { 3 - 5 } & & Prime & $\mathbf{1}^{\text {st }}$ boost & $\mathbf{2}^{\text {nd }}$ boost & \\
\hline rMVA-mep & $10^{5} \mathrm{TCID}_{50}$ & $<5$ & $<8$ & $20 \pm 1.33$ & $60^{c}$ \\
& $10^{6} \mathrm{TCID}_{50}$ & $<7$ & $9 \pm 1.33$ & $33 \pm 2.33^{*}$ & $85^{c}$ \\
& $10^{7} \mathrm{TCID}_{50}$ & $<8$ & $18 \pm 2.33^{*}$ & $56 \pm 1.01^{* *}$ & $100^{c}$ \\
Inactivated JEV vaccine & $9 \pm 1.33^{*}$ & $29 \pm 1.02^{* *}$ & $58 \pm 2.01^{* *}$ & $100^{c}$ \\
EDIII & $8 \pm 2.00^{*}$ & $28 \pm 1.99^{* *}$ & $56 \pm 1.99^{* *}$ & $100^{c}$ \\
rMEP & $9 \pm 1.01^{*}$ & $27 \pm 2.01^{* *}$ & $57 \pm 1.99^{* *}$ & $100^{c}$ \\
Wild MVA & & - & - & - & 0 \\
PBS $^{\text {b }}$ & & - & - & - & 8.33 \\
\hline
\end{tabular}

a Prime-boost-boost vaccinations (days 0,14 and 28) with rMVA-mep were carried out. On day 14 (prime), day 28 (first boost) and day 42 (second boost) sera were taken and analyzed. 50\% plaque reduction neutralizing titer (PRNT50) was shown as the geometrical reciprocal of the sera dilution resulting in a $50 \%$ reduction.

${ }^{\mathrm{b}}$ No JEV-neutralizing antibodies were detectable in 1:2 diluted sera of the mice in the PBS and wild MVA groups. $n=12$, values represent the mean \pm standard error. Standard group is $\mathrm{rMVA} / \mathrm{M}\left(10^{5} \mathrm{TCID}_{50}\right)$.

* Significance $\left[p<0.05\right.$ to animals immunized with $\left.r M V A / M\left(10^{5} \mathrm{TCID}_{50}\right)\right]$. **Significance $\left[p<0.01\right.$ to animals immunized with $\left.\mathrm{rMVA} / \mathrm{M}\left(10^{5} \mathrm{TCID}_{50}\right)\right]$.

c Significance ( $p<0.05$ to $8.33 \%)$.

in dose-dependent manner, and the highest level of immune responses was observed from the groups immunized with $10^{7} \mathrm{TCID}_{50}$ rMVA-mep among the experimental three concentrations. Our paper proved that in the mouse model experiments, live rMVA-mep could elicit strongly humoral and cellular immune responses. Unexpectedly, expect for humoral immune responses, recombinant ED3 and inactivated JE vaccine also could induce cellular immune responses. Additionally, the optimum concentration of live rMVA-mep to trigger the maximum immune responses during the immunization is unidentified, which need to be further verified.

In this paper, it was found that mice immunized with rMVA-mep produced the high level of the antibody production with the major antibody subtype IgG1 and the minor antibody subtype IgG2a. Our data showed that rMVA-mep immunization could trigger the strongly cytokine productions in which the levels of IFN- $\gamma$ were higher than that of IL-4. It has been reported that IgG1 is believed to indicate a humoral immune response, whereas IgG2a is indicative of a cellular response [40]. Furthermore, cytokines play vital roles on various immune responses. IFN- $\gamma$, the representative factor of Th1 type immune response, is produced by stimulated $\mathrm{T}$ cells and has important immunomodulatory effects [41]. IL-4 can promote B-cell differentiation and enhance the production of antibodies by sensitized B cells [42], which is the representative factor of Th2 type immune response. These results suggested that rMVA-mep could induce both the humoral and cellular immune responses. It was proved that the rMVA-mep vaccination produced strong antibody response in dose-dependent manner, and a mixed Th1/Th2 response in a mouse model.

These findings are consistent with previous studies that rMEP could induce fine immune responses. Although the levels of the neutralizing antibodies from mice immunized with three concentrations of rMVA-mep were lower than that of inactivated JEV vaccine, EDIII and rMEP controls at the prime and $1^{\text {st }}$ boost immunizations, the level of the neutralizing antibodies from mice immunized with $10^{7} \mathrm{TCID}_{50}$ rMVA-mep was almost equal to that of these controls at the $2^{\text {nd }}$ boost immunization. Also, the levels of the neutralizing antibodies from mice immunized with three concentrations of rMVA-mep were increased in dose-dependent manner. It was found that the levels of the neutralizing antibodies from mice immunized with three concentrations of rMVA-mep were increased with three time immunization. Furthermore, it was observed that $10^{7} \mathrm{TCID}_{50}$ rMVA-mep vaccination elicited high-level neutralizing antibody and protection against JEV challenge. As for the duration of neutralizing antibodies, it was required to be further investigated. These results suggested that rMVA-mep might be an attractive candidate vaccine for preventing against JEV infection.

In conclusion, we first constructed the recombinant viruses (rMVA-mep) expressing recombinant multiepitope peptide (rMEP) from JEV. It was demonstrated that rMVA-mep vaccination can elicit both humoral and cellular immune responses in a mouse model, such as antibody and cytokines productions increase, and protection response against JEV challenge. These results suggested recombinant MVA/M might be an attractive candidate vaccine for JEV infection.

\section{Competing interests}

The authors declare that they have no competing interests.

\section{Authors' contributions}

FJW and XLF carried out most of the experiments and wrote the manuscript. QSZ, HYH, BZ, XDL, RP, JZ, WLD, QTL and PYC helped with the experiments. $\mathrm{RBC}$ designed the experiments and revised the manuscript. All of the authors read and approved the final version of this manuscript.

\section{Acknowledgments}

This work was supported by the National Special Research Programs for Non-Profit Trades, Ministry of Agriculture (No. 200803015 and No.

201203082) and the National Special Research Programs for Cultivating New Transgenic Organisms, Ministry of Agriculture (NO.2009ZX08006-007B), and A Project Funded by the Priority Academic Program Development of Jiangsu Higher Education Institutions (PAPD).

\section{Author details}

${ }^{1}$ Key Laboratory of Animal Diseases Diagnosis and Immunology, Ministry of Agriculture, Nanjing Agricultural University, Nanjing 210095, China. ${ }^{2}$ National Veterinary Biological Medicine Engineering Research Center, Nanjing 210014, People's Republic of China. ${ }^{3}$ Veterinary Office, Gansu Agriculture and Animal Husbandry Department, Lanzhou 730000, People's Republic of China.

${ }^{4}$ Veterinary Medicine, Yangzhou University, Yangzhou 225009, China. 
Received: 2 November 2011 Accepted: 6 July 2012

Published: 17 September 2012

\section{References}

1. Shope RE: Medical significance of togaviruses: an overview of diseases caused by togaviruses in man and in domestic and wild vertebrate animals. In The togaviruses. Edited by Schlesinger RW. New York: Academic; 1980:47-82.

2. Monath TP: Pathobiology of the flaviviruses. In The Togaviridae and Flaviviridae. Edited by Schlesinger S, Schlesinger MJ. New York: Plenum Press; 1986:375-440.

3. Scherer WF, Moyer J, Izumi T, Gresser I, McCown J: Ecologic studies of Japanese encephalitis virus in Japan: $\mathrm{Vl}$, Swine in fection. AmJTrop Med Hyg 1959, 8:698-706.

4. Konno J, Endo K, Agatsuma H, Ishida N: Cyclic outbreaks of Japanese encephalitis among pigs and humans. Am J Epidemiol 1966, 84(2):292-300.

5. Oya A: The role of mammals as primary and supplementary hosts. Jpn J Med Sci Biol 1967, 20(Suppl):26-30.

6. Vythilingam I, Oda K, Mahadevan S, Abdullah G, Thim CS, Hong CC, Vijayamalar B, Sinniah M, Igarashi A: Abundance, parity, and Japanese encephalitis virus infection of mosquitoes (Diptera: Culicidae) in Sepang District, Malaysia. J Med Entomol 1997, 34:257-262.

7. Tsai TF, Chang GF, Yu YX: Japanese encephalitis vaccines. In Vaccines. 3rd edition. Edited by Stanley AP, Oreustein WA. Philadelphia, USA: Saunders; 1999:672-710.

8. Plesner AM: Allergic reactions to Japanese encephalitis vaccine. Immunol. Allergy Clin. North Am. 2003, 23:665-697.

9. Olszewska W, Steward MW: Nasal delivery of epitope-based vaccines. Advanced Drug Delivery Reviews 2001, 51:161-171.

10. Kimura-Kiroda J, Yasui K: Protection of mice against Japanese encephalitis virus by passive administration with monoclonal antibodies. J Immunol 1988, 141:3606-3610.

11. Mason PW, Pincus S, Fournier MJ, Masom TL, Shope RE, Paoletli E: Japanese encephalitis virus wmcinia recombinants produce particle form of the structural membrane proteins and induce high levels of protection against lethal JEV infection. Virology 1991, 180:294-305.

12. Murali-Krishna K: Ravi VR, Manjunath: Protection of adult but not newborn mice against lethal intracerebral challenge with Japanese encephalitis virus by adoptively transferred virus-specific cytotoxic T lymphocytes: requirement for L3T4+ T cells. J Gen Virol 1996, 77(Pt. 4):705-714.

13. Kaur R, Sachdeva G, Vrati S: Plasmid DNA immunization against Japanese encephalitis virus: immunogenicity of membrane-anchored and secretory envelope protein. J Infect Dis 2002, 185:1-12.

14. Dewasthaly S, Ayachit VM, Sarthi SA, Gore MM: Monoclonal antibody raised against envelope glycoprotein peptide neutralizes Japanese encephalitis virus. Arch Virol 2001, 146:1427-1435.

15. Kolaskar AS, Kulkarni-Kale U: Prediction of three-dimensional structure and mapping of conformational epitopes of envelope glycoprotein of Japanese encephalitis virus. Virology 1999, 261:31-42.

16. Seif SA, Morita K, Matsuo S, Hasebe F, Igarashi A: Finer mapping of neutralizing epitope(s) on the C-terminal of Japanese encephalitis virus E-protein expressed in recombinant Escherichia coli-system. Vaccine 1995, 13:1515-1521.

17. Sette A, Fikes J: Epitope-based vaccines: an update on epitope identification, vaccine design and delivery. Curr Opin Immunol 2003, 15:461-470

18. Sette A, Newman M, Livingston B, McKinney D, Sidney J, Ishioka G, Tangri S, Alexander J, Fikes J, Chesnut R: Optimizing vaccine design for cellular processing, MHC binding and TCR recognition. Tissue Antigens 2002, 59:443-451

19. Sette A, Livingston B, McKinney D, Appella E, Fikes J, Sidney J, New M, Chesnut $R$ : The development of multi-epitope vaccines: epitope identification, vaccine design and clinical evaluation. Biologicals 2001, 29:271-276.

20. Sutter G, Wyatt $L S$, Foley PL: A recombinant vector derived from the host range-restricted and highly attenuated MVA strain of vaccinia virus stimulates protective immunity in mice to influenza virus. Vaccine 1994, 12:1032-1040.

21. Stittelaar K, Wyatt LS, de Swart RL: Protective immunity in macaques vaccinated with a modified vaccinia virus Ankara-based measles virus vaccine in the presence of passively acquired antibodies. J Virol 2000, 74:4236-4243

22. Hirsch VM, Fuerst TR, Sutter G: Patterns of viral replication correlate with outcome in simian immunodeficiency virus (SIV)-infected macaques: effect of prior immunization with a trivalent SIV vaccine in modified vaccinia virus Ankara. J Virol 1996, 70:3741-3752.

23. Carroll MW, Overwijk WW, Chamberlain S: Highly attenuated modified vaccinia virus Ankara (MVA) as an effective recombinant vector: a murine tumor model. Vaccine 1997, 15:387-394.

24. Espenschied J, Lamont J, Longmate J: CTLA-4 Blockade Enhances the Therapeutic Effect of an Attenuated Poxvirus Vaccine Targeting p53 in an Established Murine Tumor Modes. J Immunol 2003, 170:3401-3407.

25. Rosenberg SA: Cancer vaccines based on the identification of the genes encoding cancer regression antigens. Immunol Today 1997, 18:175-182.

26. Schneider J, Gilbert SC, Blanchard TJ, Hanke T, Robson KJ, Hannan CM: Enhanced immunogenicity for CD8+ T cell induction and complete protective efficacy of malaria DNA vaccination by boosting with modified vaccinia virus Ankara. Nat Med 1998, 4:397-402.

27. Zavala F, Rodrigues M, Rodriguez D, Rodriguez JR, Nussenzweig RS, Esteban M: A striking property of recombinant poxviruses: efficient inducers of in vivo expansion of primed CD8 (+) T cells. Virology 2001, 280:155-159.

28. Ramirez JC, Gherardi MM, Rodriguez D: Attenuated modified vaccinia virus Ankara can be used as an immunizing agent under conditions of preexisting immunity to the vector. J Virol 2000, 74:7651-7655.

29. Wei J-, Huang Y-, Zhong D-, Kang L, Ishag H, Mao X, Cao R-, Zhou B, Chen $P$-: Design and evaluation of a multi-epitope peptide against Japanese encephalitis virus infection in BALB/c mice. Biochem Biophys Res Commun 2010, 96:787-792.

30. Ho CY: Lau Clara BS, Kim CF, Leung KN, Fung KP, Tse TF, Chan Helen HL, Chow Moses SS: Differential effect of Coriolus versicolor (Yunzhi) extract on cytokine production by murine lymphocytes in vitro. Int Immunopharmacol 2004, 4:1549-1557.

31. Stuart N: Isaacs: Vaccinia Virus and Poxvirology: Methods and Protocols in Methods in Molecular Biology, vol. 269. Totowa, New Jersey: Humana Press Inc; 2004.

32. Ding J, Lu Y, Chen Y: Candidate multi-epitope vaccines in aluminium adjuvant induce high levels of antibodies with predefined multi-epitope specificity against HIV-1. FEMS Immunol Med Microbiol 2000, 29:123-127.

33. Wu SC, Yu CH, Lin CW, Chu IM: The domain III fragment of Japanese encephalitis virus envelope protein: mouse immunogenicity and liposome adjuvanticity. Vaccine 2003, 21:2516-2522.

34. Vrati S, Agarwal V, Malik P, Wani SA, Saini M: Molecular characterization of Indian isolate of Japanese encephalitis virus that shows an extended lag phase during growth. J Gen Virol 1999, 80:1665-1671.

35. Hamano M, Lim CK, Takagi H, Sawabe K, Kuwayama M, Kishi N: Detection of antibodies to Japanese encephalitis virus in the wild boars in Hiroshima prefecture, Japan. Epidemiol Infect 2007, 135(6):974-977.

36. Kaur $R$, Vrati S: Development of a recombinant vaccine against Japanese encephalitis. J Neurovirol 2003, 9:421-431.

37. Lowenadler B, Lycke N, Svanholm C, Svennerholm AM, Krook K, Gidlund M: $T$ and $B$ cell responses to chimeric proteins containing heterologous $T$ helper epitopes inserted at different positions. Mol Immunol 1992, 29:1185-1190

38. Shirai M, Pendleton CD, Ahlers J, Takeshita T, Newman M, Berzofsky JA: Helper-cytotoxic T lymphocyte (CTL) determinant linkage required for priming of anti-HIV CD8+ CTL in vivo with peptide vaccine constructs. J Immunol 1994, 152:549-556.

39. Cardin RD, Brooks JW, Sarawar SR, Doherty PC: Progressive loss of CD8+ T cell-mediated control of a gamma-herpesvirus in the absence of CD4+ $T$ cells. J Exp Med 1996, 184:863-871.

40. Hauge S, Madhun A, Cox RJ, Haaheim LR: Quality and kinetics of the antibody response in mice after three different low-dose influenza virus vaccination strategies. Clin Vaccine Immunol 2007, 14:978-983.

41. Farrar MA, Schreiber RD: The molecular cell biology of interferon- $\gamma$ and its receptor. Annu Rev Immunol 1993, 11:571-611.

42. Belardelli F: Role of interferons and other cytokines in the regulation of the immune response. APMIS 1995, 103:161-179.

\section{doi:10.1186/1743-422X-9-204}

Cite this article as: Wang et al: Multiple linear epitopes (B-cell, CTL and Th) of JEV expressed in recombinant MVA as multiple epitope vaccine induces a protective immune response. Virology Journal 2012 9:204. 